\title{
ORIGINAL ARTICLE \\ The influence of ankle joint mobility when using an orthosis on stability in patients with spinal cord injury: a pilot study
}

\author{
M Arazpour ${ }^{1}$, MA Bani ${ }^{1}$, SW Hutchins ${ }^{2}$, S Curran ${ }^{3}$ and MA Javanshir ${ }^{4}$
}

Objectives: Perceived risk of falling is an important factor for people with spinal cord injury (SCI). This study investigated the influence of ankle joint motion on postural stability and walking in people with $\mathrm{SCl}$ when using an orthosis.

Methods: Volunteer subjects with $\mathrm{SCl}(n=5)$ participated in this study. Each subject was fitted with an advanced reciprocating gait orthosis (ARGO) equipped with either solid or dorsiflexion-assist type ankle-foot orthosis (AFOs) and walked at their self-selected speed along a flat walkway to enable the comparison of walking speed, cadence and endurance. A force plate system and a modified Falls Efficacy Scale (MFES) were utilized to measure postural sway and the perceived fear of falling, respectively.

Results: There were significant differences in the mean MFES scores between two types of orthosis $(P=0.023)$. When using two crutches, there was no significant difference in static standing postural sway in the medio-lateral $(\mathrm{M} / \mathrm{L})$ direction $(P=0.799)$, but significant difference in the antero-posterior $(A / P)$ direction $(P=0.014)$. However, during single crutch support, there was a significant difference in both $\mathrm{M} / \mathrm{L}(P=0.019)$ and $\mathrm{A} / \mathrm{P}(P=0.022)$ directions. Walking speed $(7 \%)$ and endurance $(5 \%)$ significantly increased when using the ARGO with dorsi flexion assisted AFOs. There was no significant deference between two types of orthoses in cadence $(P=0.54)$.

Conclusions: Using an ARGO with dorsiflexion-assisted AFOs increased the fear of falling, but improved static postural stability and increased walking speed and endurance, and should therefore be considered as an effective orthosis during the rehabilitation of people with $\mathrm{SCl}$.

Spinal Cord (2013) 51, 750-754; doi:10.1038/sc.2013.78; published online 30 July 2013

Keywords: advanced reciprocating gait orthoses; postural stability; walking; rehabilitation; spinal cord injury; paraplegia

\section{INTRODUCTION}

People with spinal cord injury (SCI) use orthoses such as the hip knee ankle-foot orthosis, The Walkabout and Primewalk orthoses, the advanced reciprocating gait orthosis (ARGO) and the isocentric reciprocating gait orthosis for standing and walking purposes. ${ }^{1}$ Positive physiological and psychological effects have been demonstrated in SCI subjects when undertaking standing and walking activities, such as a reduction in the incidence of osteoporosis, contractures, atrophy of lower limb muscles, bed sores and fractures. Improvements in self-esteem and social communication have also been reported. ${ }^{2}$

An orthosis is used to facilitate mobility and independence for people with SCI who are otherwise confined to wheelchair use. Independent standing and gait training via orthoses are important for the ability to undertake activities of daily living in such patients. ${ }^{3}$ Postural stability in people with SCI, when ambulating is achieved by using both an orthosis and a walking aid, such as a crutch or walking frame. The existence of a mechanism within the orthosis linking both sides (for example, the reciprocal link in an isocentric reciprocating gait orthosis) also provides stability. The amount of structural rigidity inherent in the orthosis design gives an overall support and extrinsic stability. In addition, people with SCI normally adopt a 'C-type' posture (that is, adopt a long sagittal plane spinal curve with the apex pointing forwards) via hyperextension of the lumbar and thoracolumbar spine and by extending the hip joints in order to alter the position of the torso over the lower limbs in the sagittal plane; especially during initiation of motion. During such posturing, the anterior femoral ligament and trunk muscles with the addition of support through the upper limbs via walking aids, all provide stability. ${ }^{3}$

Lack of postural control is regarded as one of the main reasons for causing a fear of falling among the people with SCI during their rehabilitation programs that are designed to improve their ability to walk and stand. ${ }^{4,5}$ Brotherton et al. ${ }^{6}$ stated that falls by individuals with SCI often occurred in the home, especially during the day. The incidence of fractures has been reported as being $18 \%$; (5-6\% greater than that experienced by healthy older adults). ${ }^{7,8}$ In addition, the incidence rate for falls in people with SCI is $75 \%$, which is higher than the incidence reported for healthy subjects aged 65 and older $(35 \%),{ }^{7,9}$ and is also higher than those reported for subjects with neurological disease resulting in peripheral neuropathy $(50 \%)$ or Parkinson's disease (38-62\%). ${ }^{10-12}$ These figures highlight the need for an adequate evaluation and performance in walking and standing for people with SCI.

${ }^{1}$ Orthotics and Prosthetics Department, University of Social Welfare and Rehabilitation Science, Tehran, Iran; ${ }^{2}$ University of Salford, IHSCR, Faculty of Health and Social Care, University of Salford, Salford, UK; ${ }^{3}$ Cardiff School of Health Sciences, Llandaff Campus, Cardiff Metropolitan University, Cardiff, Wales, UK and ${ }^{4}$ Orthotics and Prosthetics Department, Iran University of Medical Sciences, Tehran, Iran

Correspondence: MA Javanshir, Department of Orthotics and Prosthetics, Iran University of Medical Science, Rehabilitation Faculty, Mirdamad Blvd, Shahnazari Street, Tehran, Iran.

E-mail: M.ali_javanshir@yahoo.com

Received 1 April 2013; revised 22 June 2013; accepted 25 June 2013; published online 30 July 2013 
Balance and walking impairment have been associated with an increase in the prevalence of falls and subsequent injuries in people with SCI during walking ${ }^{13}$ and has proven to be the main problem experienced by SCI patients. ${ }^{6,14}$ Brotherton et al., ${ }^{6}$ stated that postural control has an essential role in the prevention of falls, as patients with good postural control demonstrate better walking and standing performance. Lin et al. ${ }^{15}$ evaluated the kinematics, kinetics and electromyography associated with postural control during static stance when people with SCI voluntarily let go of their walking frame. The resulting instability caused a rapid reach-and-grasp balance reaction in these individuals with thoracic level of injury. However, it was demonstrated that the resultant sudden increase of upper back extensor muscle activity did not provide enough intrinsic power to maintain balance. Baardman et al. ${ }^{16}$ evaluated six male subjects with SCI and analyzed and compared their static standing stability and their ability to handle balance disturbances when supported by a standard ARGO or an ARGO with the Bowden cable removed. They hypothesized that due to the increased crutch forces measured when standing with the ARGO with the cable removed, the reciprocal link must have been instrumental in decreasing the effort required to stand while being supported via elbow crutches. Ahmadi Bani et al. ${ }^{17}$ in an evaluation of an ARGO incorporating either solid AFOs or dorsiflexion-assist AFOs demonstrated that an ARGO with dorsiflexion-assist AFOs increased walking speed and step length plus ankle joint range of motion, but did not assess postural stability.

Although the position and orientation of the center of pressure is an important factor in stability during static stance, ${ }^{18}$ the ankle joint sagittal plane motion and position may also have an important role in paraplegic standing and walking. The aim of this study was therefore to investigate the effect of sagittal plane ankle joint motion on stability and the perceived risk of falling during ambulation in patients with SCI. The hypothesis was, that by provision of a dorsiflexion-assist mechanism at the ankle level in an ARGO, parameters relevant to static and dynamic stability and perceived risk of falls would be altered compared with when walking with an ARGO within which the ankle joints were fixed in position.

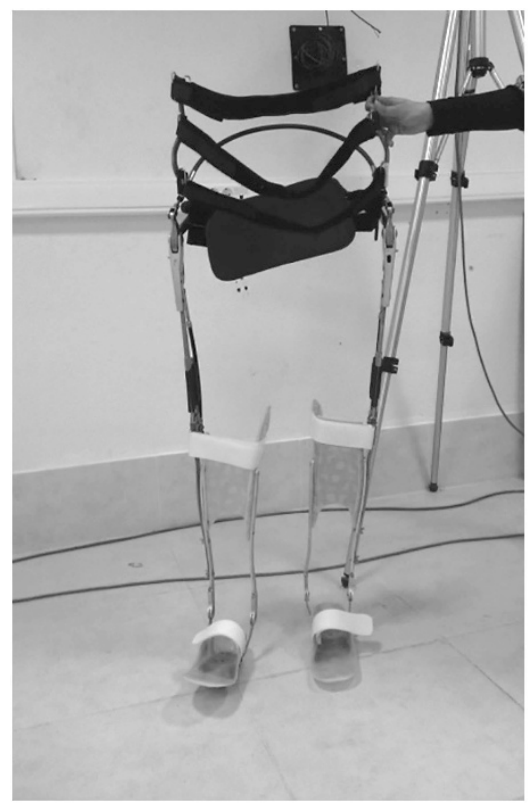

Figure 1 The advanced reciprocal gait orthoses used in this study.

\section{MATERIALS AND METHODS}

\section{Subjects}

Five subjects with SCI $(n=5)$ were recruited to the study by convenience sampling. Inclusion criteria for this study included the ability to walk with an ARGO for a minimum of $50 \mathrm{~m}$ independently when performing a crutchassisted reciprocal four-point gait pattern, the ability to stand for at least $90 \mathrm{~s}$ independently with the orthosis, and were required to have completed a 12-week gait training rehabilitation program before being included. The presence of a head injury, previous lower limb or upper limb fractures, pressure sores or any psychiatric comorbidities were considered as an exclusion criteria.

\section{Orthotic intervention}

Each patient was supplied with an ARGO specifically made for them, which was fitted with either solid AFOs or with dorsiflexion-assist ankle joints. The ARGOs were manufactured from casts of the lower limbs of people with SCI by an experienced orthotist.

\section{Outcome Measures}

The modified falls efficacy scale. This scale was utilized in this study and was completed following completion of the test conditions. The modified Falls Efficacy Scale (MFES) uses a visual analog scale to grade 14 activities, whereby each activity is rated from 1 (lack of confidence and high fear of falling) to 10 (high confidence and low fear of falling). All 14 activities were analyzed in this study.

\section{Procedure}

Volunteer subjects were randomly assigned to either the ARGO with solid AFOs or the ARGO which used dorsiflexion-assist AFOs. For randomization, the patients chose either ' $\mathrm{A}$ ' or ' $\mathrm{B}$ '. In condition ' $\mathrm{A}$ ', the subjects walked first using the ARGO with solid AFOs, and in condition 'B', they first walked with the ARGO with dorsiflexion-assist AFOs. The volunteer subjects with SCI received further gait training with the orthoses ranging from 6 to 10 weeks according to the level and severity of the injury. Strengthening of the upper body muscles, training in transferring, donning and doffing of the orthosis, balancing in a standing position and walking training on flat surfaces were all included in the gait training program. On completion of the gait training with the orthoses, each patient could walk with either orthosis independently without falling using a walking aid. Figure 1 shows the two versions of the ARGO used in this study.

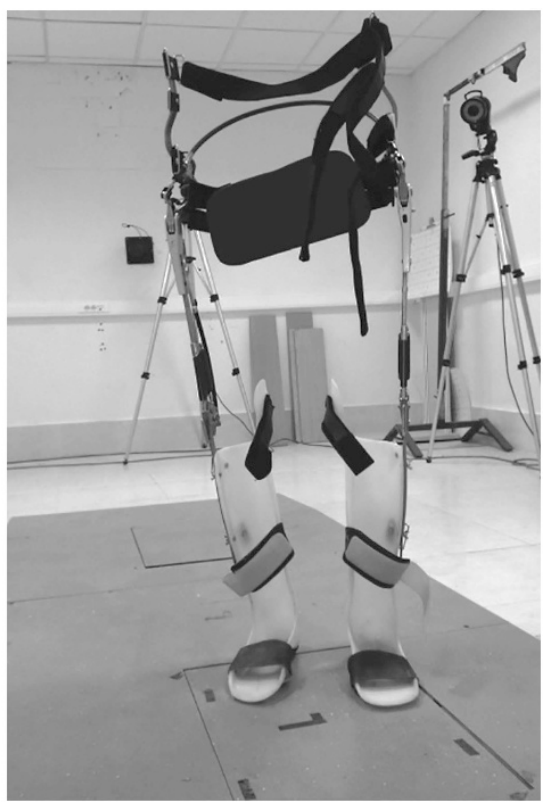


When the volunteer subjects used the solid ankle-foot orthosis test condition first, the cosmetic AFO sections were subsequently removed and replaced with the dorsiflexion-assist conventional AFO lower leg sections. This enabled the same ARGO superstructure to be used for each test condition. When they used the dorsiflexion-assisted AFO first, they again removed the conventional AFO section with $4 \mathrm{~mm}$ copolymer polypropylene thermoplastic solid AFOs.

Patients were instructed to walk using each orthotic test condition with the aid of elbow crutches for a total of $3 \mathrm{~min}$. Both walking speed $\left(\mathrm{ms}^{-1}\right)$ and cadence (steps per min) were recorded during the second minute of each 3 min walking session using a hand-held stopwatch. The maximum distance the patient was able to walk without resting within the 3-min period was taken as the endurance measure for each walking activity. A 1-h rest time was allowed between the two test conditions (that is, the two types of orthoses).

Postural sway assessment was performed on a different day to mitigate the effects of tiredness. Each patient stood on a force plate system (Kistler 9286BA, Winterthur, Switzerland) which was used to measure the effect of postural sway on the center of pressure position on the force plate. Participants stood at a position approximating to the center of the force plate when wearing either the ARGO with solid AFOs or the ARGO with dorsiflexion-assist AFOs randomly. Measurements were taken when the patient used two elbow crutches and also when using just one elbow crutch on their dominant side. During evaluation, the participants were asked to fix their eyes focused straight ahead at a distance of $3 \mathrm{~m}$ for $30 \mathrm{~s}$ and were instructed not to speak during the test. Three measurements were performed and the average value was calculated for statistical analysis.

The Human Ethical Committee of University of Social Welfare and Rehabilitation Sciences gave approval to perform the study. The study protocol was explained to all volunteer subjects and a consent form was signed by all participants.

\section{Statistical analysis}

The outcome measures for this study were the mean postural sway of the center of pressure in the medio-lateral and the antero-posterior $(\mathrm{A} / \mathrm{P})$ direction, the MFES scores, walking speed, cadence and endurance. Normality

Table1 Patients information who participated in this study

\begin{tabular}{llcclcc}
\hline $\begin{array}{l}\text { Patient } \\
\text { number }\end{array}$ & Sex & $\begin{array}{c}\text { Age } \\
\text { (years) }\end{array}$ & $\begin{array}{c}\text { Weight } \\
(\mathrm{kg})\end{array}$ & $\begin{array}{c}\text { Level of } \\
\text { injury }\end{array}$ & $\begin{array}{c}\text { ASIA } \\
\text { score }\end{array}$ & $\begin{array}{c}\text { Time since injury } \\
\text { (months) }\end{array}$ \\
\hline 1 & Male & 28 & 78 & T12 & B & 26 \\
2 & Male & 26 & 68 & T12 & A & 45 \\
3 & Male & 32 & 70 & T8 & B & 24 \\
4 & Female & 20 & 58 & T12 & B & 28 \\
5 & Male & 26 & 64 & T10 & A & 33 \\
\hline
\end{tabular}

Abbreviation: ASIA, American Spinal Injury Association. of data was confirmed by using the Kolmogorov-Smirnov technique. According to the normality of data, a paired $t$-test was used for analyzing the effects and differences in the selected outcome measures between the two orthoses SPSS for Windows (SPSS Inc, Chicago, IL, USA) was used for data analyzing. The significance level was set at $P \leqslant 0.05$.

\section{RESULTS}

Table 1 shows the demographic data regarding the people with SCI who volunteered for this study.

Table 2 shows mean and s.d. of the parameters investigated in this study when wearing the two orthoses. The mean MFES scores when the using the ARGO with solid AFOs and the dorsiflexion-assisted AFOs were $45.80 \pm 9.12$ and $42.80 \pm 9.73$, respectively. There was significant difference between the two types of orthosis in this parameter $(P=0.023)$.

During static dual-elbow crutch support, there was no significant difference between the two types of orthosis in the postural sway in the medio-lateral direction $(P=0.799)$, but significant difference between them in the A/P direction $(P=0.014)$. However, during single elbow crutch support, there was significant difference between two types of orthosis in postural sway in both planes (medio-lateral, $P=0.019 ; \mathrm{A} / \mathrm{P}, P=0.022)$.

Walking speed significantly increased (7\%) when using the ARGO with dorsiflexion assist AFOs compared with the ARGO with solid AFOs. There was no significant difference between the two types of orthoses in cadence $(P=0.541)$, but endurance of walking significantly increased (5\%) in using ARGO with dorsi flexion-assisted AFO compared with ARGO with solid AFO $(P=0.012)$.

\section{DISCUSSION}

This study was performed to determine the effect of wearing the same ARGO but with different lower limb designs on the amount of postural sway, the perceived fear of falling and their alteration to specific gait parameters by people with SCI. This was done to more fully understand the ramifications of adding sagittal plane motion to the ankle joints of such orthoses and the relationship between this mechanism and stability as well as its effect on gait parameters. Confidence in performing activities of daily living without falling has historically been evaluated by the MFES among others. ${ }^{19}$ The intraclass correlation coefficient of the MFES for retest reliability has been reported as being high (0.58) and internal reliability has been shown to be excellent (Cronbach's alpha $=0.97){ }^{20}$

The ability to perform activities of daily living by people with SCI are dependent on provision of orthoses which offer postural stability both when standing and walking while relying on the use of the upper

Table2 Mean and s.d. and statistical analysis of postural sway measurements, gait parameters and Modified Falls Efficacy Scale (MFES) scores in walking with the two types of orthosis

\begin{tabular}{|c|c|c|c|c|c|c|c|c|}
\hline & \multicolumn{4}{|c|}{ Postural sway measurements } & \multicolumn{3}{|c|}{ Gait parameters } & \multirow[t]{3}{*}{ MFES score } \\
\hline & \multicolumn{2}{|c|}{ Dual hand support } & \multicolumn{2}{|c|}{ Single hand support } & \multirow{2}{*}{$\begin{array}{l}\text { Speed } \\
\left(m s^{-1}\right)\end{array}$} & \multirow{2}{*}{$\begin{array}{c}\text { Cadence } \\
\text { (steps per min) }\end{array}$} & \multirow{2}{*}{$\begin{array}{c}\text { Endurance } \\
\text { (m) }\end{array}$} & \\
\hline & $\begin{array}{l}\text { Medio-lateral } \\
\text { direction }(\mathrm{mm})\end{array}$ & $\begin{array}{l}\text { Antero-posterior } \\
\text { direction }(\mathrm{mm})\end{array}$ & $\begin{array}{l}\text { Medio-lateral } \\
\text { direction }(\mathrm{mm})\end{array}$ & $\begin{array}{l}\text { Antero-posterior } \\
\text { direction }(\mathrm{mm})\end{array}$ & & & & \\
\hline Walking with ARGO with solid AFO & $38.2 \pm 2.86$ & $33.6 \pm 2.30$ & $42.4 \pm 3.04$ & $43.6 \pm 3.84$ & $0.27 \pm .03$ & $20.6 \pm 2.19$ & $93.2 \pm 14.61$ & $45.8 \pm 9.12$ \\
\hline $\begin{array}{l}\text { Walking with ARGO with dorsi } \\
\text { flexion-assisted AFO }\end{array}$ & $38.4 \pm 1.67$ & $30.2 \pm 3.63$ & $45.0 \pm 2.91$ & $38.6 \pm 1.94$ & $0.29 \pm .03$ & $21.0 \pm 3.08$ & $98.2 \pm 15.27$ & $42.8 \pm 9.73$ \\
\hline$P$-value & 0.799 & 0.014 & 0.019 & 0.022 & 0.011 & 0.541 & 0.012 & 0.023 \\
\hline
\end{tabular}

Abbreviations: AFO, ankle-foot orthosis; ARGO, advanced reciprocating gait orthosis; MFES, modified Falls Efficacy Scale. 
limbs for additional support via walking aids. ${ }^{5,21}$ It was noted that patients had a lower fear of falling (that is, felt more safe) when using the ARGO with solid AFOs, as evidenced by the higher MFES scores, but interestingly had more postural sway in the $\mathrm{A} / \mathrm{P}$ direction compared with using the ARGO with dorsiflexion-assist AFOs during static stance with both single and double hand support. This increased postural sway may have been due to the dorsiflexion assist ankle joints allowing limited ankle motion rather than with fixed AFOs, where this would be translated into A/P motion through the AFO superstructure to the force plate. In addition, during single hand support, more lateral postural sway was detected with the dorsiflexion-assist AFOs. This would be expected due to the increased lateral control offered by ankle blocking solid AFOs. The findings of this study are supported by the observations made by John et al. ${ }^{5}$ who demonstrated a statistically significant negative correlation between the MFES scores and the postural sway measurements.

It is not clear whether there is a causal link between the reduced postural sway in the $\mathrm{A} / \mathrm{P}$ direction afforded by standing with dorsiflexion-assist AFOs and the increase in walking speed and endurance demonstrated during the walking trials compared with the device with fixed solid cosmetic AFOs. The dorsiflexion assist function at the ankle may have afforded more toe clearance during swing phase of gait but at the expense of reduced confidence demonstrated by the MFES scores.

The findings presented here may have an important role in the rehabilitation of individuals with paraplegia. The use of the ARGO associated with dorsiflexion-assisted AFO to walk has a potential to reduce the energy consumption based on increased endurance of patients to walk with this type of orthosis compared with the ARGO with solid AFO. However, this needs to be confirmed in a future study. Patients had less fear of falling sense when using ARGO with solid thermoplastic AFOs. This may have been due to the fact that with solid AFOs, they would have had more confidence that their ankle positions would not alter and be more stable.

In this present study, walking speed and endurance increased significantly in using the ARGO which allowed ankle dorsiflexion. Although this would be advantageous for SCI subjects, further investigations are needed to determine the correlation between ankle joint range of motion, static postural stability and the MFES scores in addition to resulting energy consumption levels to ascertain whether providing ankle motion via orthotic ankle joints will be a feasible and safe option. It may be that an increased walking speed when ambulating with an orthotic device increases the fear of falling in people with SCI, especially if the ankles are not fixed in position with a solid AFO.

As in previous studies within this field, people with higher levels of SCI demonstrated reduced walking speed and endurance compared with patients with lower levels of injury. Participants with lesion levels in T8 and T10 had more instability than their counterparts with lesion levels in T12 during static postural stability testing. However, John et al. ${ }^{5}$ demonstrated a statistically insignificant positive correlation between lesion level and postural sway when using KAFOs.

In this present study, people with SCI had significantly superior balance maintenance capability in medio-lateral and antero-posterior directions when using the ARGO with dorsiflexion assist than when using the ARGO with solid AFOs. Stability of the pelvis and hip joints associated with movable ankle joint in dorsiflexion direction may have contributed to this result. When using alternative orthoses which do not incorporate a pelvic girdle and hip joints (for example, the walkabout orthosis and conventional KAFOs), the iliofemoral ligament and hyperextension of the hips provide stability during paraplegic standing. When people with SCI use an ARGO, movement of the pelvis and hip joint in a standing position is still possible but is limited in all directions by the pelvic girdle or corset. An articulating orthotic ankle joint when compared with a solid AFO may be having an important role in this point. Abe et al. ${ }^{22}$ reported that the RGO was superior in static balance compared with the walkabout and double KAFOs. Baardman et al. ${ }^{16}$ demonstrated that the reciprocal hip joint link in the ARGO provided better stability compared with an ARGO without the reciprocal link removed. Using the reciprocal hip joint link in the ARGO may therefore facilitate a reduction of upper body effort required for standing under functional conditions in SCI patients.

\section{CONCLUSIONS}

The present study demonstrated that using an ARGO with dorsiflexion-assisted AFOs increased the fear of falling, but improved postural stability and increased walking speed and endurance in people with SCI compared with using an ARGO with solid AFOs. ARGO with dorsiflexion-assisted AFO should be consider as an effective orthosis in postural control during rehabilitation of walking and standing of individuals with paraplegia, but more research is needed to determine why during ambulation this type of device produced a small but significant increase in the perceived risk of falling in such patients. This study was limited by the small number of participants, and a larger study is needed to confirm that these results are indicative of the SCI population more generally. An ABAB approach would also yield more information rather than the use of one orthosis followed by the other, and the training effect may then have been minimized. In addition, there is a need to link these types of results to the energy cost of walking and to define the limit to which ankle motion may be afforded to such patients without producing an increased fear of falling.

\section{DATA ARCHIVING}

There were no data to deposit.

\section{CONFLICT OF INTEREST}

The authors declare no conflict of interest.

1 Arazpour M, Bani MA, Hutchins SW. Reciprocal gait orthoses and powered gait orthoses for walking by spinal cord injury patients. Prosthet Orthot Int 2012; 37: $14-21$.

2 Leung AKL, Wong AFY, Wong ECW, Hutchins SW. The physiological cost index of walking with an isocentric reciprocating gait orthosis among patients with T12-L1 spinal cord injury. Prosthet Orthot Int 2009; 33: 61-68.

3 Somers M. Spinal cord injury: functional rehabilitation, 3rd ed Prentice Hall, 2009

4 Kannus P, Niemi S, Palvanen M, Parkkari J. Continuously increasing number and incidence of fall-induced, fracture-associated, spinal cord injuries in elderly persons. Arch Intern Med 2000; 160: 2145-2149.

5 John LT, Binu Cherian B, Babu A. Postural control and fear of falling in persons with low-level paraplegia. J Rehab Res Dev 2010; 47: 497-502.

6 Brotherton SS, Krause JS, Nietert PJ. Falls in individuals with incomplete spinal cord injury. Spinal Cord 2006; 45: 37-40.

7 Tinetti ME, Speechley M, Ginter SF. Risk factors for falls among elderly persons living in the community. N Eng/ J Med 1988; 319: 1701-1707.

8 Gryfe C, Amies A, Ashley M. A longitudinal study of falls in an elderly population: I. Incidence and morbidity. Age Ageing 1977; 6: 201-210.

9 Prudham D, Evans JG. Factors associated with falls in the elderly: a community study. Age Ageing 1981; 10: 141-146.

10 Richardson JK, Hurvitz EA. Peripheral neuropathy: a true risk factor for falls. J Gerontol A Biol Sci Med Sci 1995; 50: M211-M215.

11 Stack E, Ashburn A. Fall events described by people with Parkinson's disease: implications for clinical interviewing and the research agenda. Physiother Res Int 1999; 4: 190-200 
12 Hirsch MA, Toole T, Maitland CG, Rider RA. The effects of balance training and highintensity resistance training on persons with idiopathic Parkinson's disease. Archi Phys Med Rehab 2003; 84: 1109-1117.

13 Krause JS. Factors associated with risk for subsequent injuries after traumatic spinal cord injury. Arch Phys Med Rehab 2004; 85: 1503-1508.

14 Brotherton SS, Krause JS, Nietert PJ. A pilot study of factors associated with falls in individuals with incomplete spinal cord injury. J Spinal Cord Med 2007; 30: 243-250.

15 Lin K, Lu T, Hsu P, Yu S, Liao W. Postural responses during falling with rapid reachand-grasp balance reaction in patients with motor complete paraplegia. Spinal Cord 2007; 46: 204-209.

16 Baardman G, IJzerman M, Hermen H, Veltink P, Boom H, Zilvold G. The influence of the reciprocal hip joint link in the Advanced Reciprocating Gait Orthosis on standing performance in paraplegia. Prosthet Orthot Int 1997; 21: 210-221.
17 Bani MA, Arazpour M, Ghomshe FT, Mousavi ME, Hutchins SW. Gait evaluation of the advanced reciprocating gait orthosis with solid versus dorsi flexion assist ankle foot orthoses in paraplegic patients. Prosthet Orthot Int 2012; 37: 161-167.

18 Middleton JW, Sinclair PJ, Smith RM, Davis GM. Postural control during stance in paraplegia: effects of medially linked versus unlinked knee-ankle-foot orthoses. Arch Phys Med Rehab 1999; 80: 1558-1565.

19 Hill KD, Schwarz JA, Kalogeropoulos AJ, Gibson SJ. Fear of falling revisited. Arch Phys Med Rehab 1996; 77: 1025-1029.

20 Parry S, Steen N, Galloway S, Kenny R, Bond J. Falls and confidence related quality of life outcome measures in an older British cohort. Postgraduate Med J 2001; 77: 103-108.

21 Umphred DA, Jewell MJ. Neurological Rehabilitation. Mosby, 2001.

22 Abe K. Comparison of static balance, walking velocity, and energy consumption with knee-ankle-foot orthosis, walkabout orthosis, and reciprocating gait orthosis in thoracic-level paraplegic patients. J Prosth Orthot 2006; 18: 87-91. 